\title{
The rigidity of the Earth's inner core
}

\author{
Keith Edward Bullen \\ Annali di Geofisica, Vol. 6, n. 1, 1953.
}

\begin{abstract}
The evidence for the existence of significant rigidity in the Earth's inner core is summarised and discussed in the light of recent work. Quantummechanical calculations, based on a Thomas-Fermi-Dirac model, suggest that an earlier estimate of $3.6 \times 10^{12} \mathrm{dyn} / \mathrm{cm}^{2}$ for this rigidity may need to be reduced by $0.5 \times 10^{12} \mathrm{dyn} / \mathrm{cm}^{2}$. The theoretical travel-times of the phase PKJKP would then need to be increased by amounts ranging from zero to 40 seconds. It is shown that the use of Gutenberg's seismic data in place of that of Jeffreys would reduce the estimated rigidity of the inner core by a further $1.6 \times 10^{12} \mathrm{dyn} / \mathrm{cm}^{2}$, approximately. It is suggested that Gutenberg's data lead to the lowest likely value for the rigidity of the inner core, namely $1.5 \times 10^{12} \mathrm{dyn} / \mathrm{cm}^{2}$, so that, on all present evidence, it is improbable that the inner core is less than about twice as rigid as steel is at zero pressure.
\end{abstract}

1. The purpose of this paper is to examine and assess, in the light of recent evidence, the theory that the Earth's inner core has a significant rigidity.

The presence of an inner core in the Earth is revealed from observations of the seismic phase PKP in the «shadow zone» for which the epicentral distance $\Delta$ lies in the range $105^{\circ}<\Delta<143^{\circ}$. Miss I.Lehmann in 1936 , followed by Gutenberg and Richter in 1938, attributed these observations to the presence of an inner core; and Jeffreys in 1939 [1939a] applied Airy's theory of diffraction near a caustic to show that the alternative theory of diffraction round the outer boundary of the central core was not capable of explaining the observations in the shadow zone. The existence of the inner core has been fairly generally accepted since this calculation of Jeffreys.

2. The work of Jeffreys [1939b] on the $P$-velocity, $\alpha$ say, in the central core favours, the view that the inner core is composite, consisting of an outer region $F$ [Bullen 1947] occupying the range $1250 \mathrm{~km}<r<1390 \mathrm{~km}$, where $r$ denotes the distance from the centre, and an inner region $G$ of radius $1250 \mathrm{~km}$; (the region $E$ refers to the outer part of the core, for which $1390 \mathrm{~km}<r<3470 \mathrm{~km}$; and the region $D$ to that part of the mantle for which $3470 \mathrm{~km}<r<5400$ $\mathrm{km}$, approx.). In the solution given by Jeffreys, the value of $\alpha$ in $F$ decreases with increase of depth from $10.44 \mathrm{~km} / \mathrm{sec}$ to $9.5 \mathrm{~km} / \mathrm{sec}$ (the latter figure corresponds to a rate of decrease of a taken arbitrarily to be linear by Jeffreys), while, in $\mathrm{G}, \alpha$ increases slowly from $11.16 \mathrm{~km} / \mathrm{sec}$ at the boundary to $11.3 \mathrm{~km} / \mathrm{sec}$ at the centre; across the boundary between $F$ and $G$, there is a discontinuous jump in a. The existence of the region $F$ in which the velocity gradient is negative is not accepted by all authors, for example Gutenberg [1951]; (the existence of $F$ rests on a hypothesis of reflection at the inner core boundary which Jeffreys considers may well be true in the light of various supporting evidence).

Gutenberg's velocity distribution, on the other hand, gives a steady increase of $\alpha$ from $8.0 \mathrm{~km} / \mathrm{sec}$ at the outer boundary of the central core to $10.1 \mathrm{~km} / \mathrm{sec}$ at $r=1250 \mathrm{~km}$, then a sharp increase in the gradient of $\alpha$, with no discontinuous change in $\alpha$ itself, resulting in a value of $\alpha$ equal to $11.2 \mathrm{~km} / \mathrm{sec}$ at about $r=1000 \mathrm{~km}$; from here to the centre, Gutenberg finds to be nearly constant.

3. Following calculations [Bullen 1940, 1942] on the pressure and density distributions in the Earth, the present writer noted in 1946 that the indicated values of $d k / d p$, where $k$ denotes the incompressibility and $p$ the pressure, were not sensibly different in the outer part of the central core from the values in the lower mantle down to a depth of $2700 \mathrm{~km}$. (This result is indicated most clearly by consideration of the relation

$$
d k / d p=1-g^{-1} d ø / d r
$$

which applies to a homogeneous material, where $\mathrm{g}$ denotes the acceleration due to gravity, $\varnothing=\alpha^{2}-4 \beta^{2} / 3$, and $\beta$ denotes the $S$-velocity). Moreover, the change in $k$ itself was so small as to be not necessarily significant. All well-established geophysical data could in fact be fitted by assuming that $k$ and $d k / d p$ change smoothly between the mantle and the core. At the same time, it was noted that the materials of the mantle and core are widely different in respect of the density $\rho$ and rigidity $\mu$. Further, in 1946 , it was widely accepted that the chemical compositions of the mantle and core are distinct, that the mantle is composed of ultrabasic rock, and the outer core of iron or nickel-iron. And, in addition, 
laboratory investigations at high pressure had shown that, in general, the differences in the compressibilities of different materials diminish with increasing pressure.

It these circumstances, the writer proposed as a trial working hypothesis [Bullen 1946, 1947, 1949a] that:

a) the compressibility of a material and its pressure gradient are independent of chemical composition at pressures and temperatures of the order of those prevailing in the Earth's deep interior (say below a depth of $1000 \mathrm{~km}$ ).

4. When the hypothesis (a) was first set down, it had not been envisaged that there would soon be a serious challenge to the view that the mantle and outer core are chemically distinct. Following work of Kronig, de Boer and Korringa [1946], however, Ramsey [1948, 1949] and the writer [Bullen 1949b], on the basis of planetary data, have held that there is a probability that the region $E$ consists essentially of a pressure modification of ultrabasic rock; (Ramsey, but not the present writer, holds that the inner core, also, is caused by pressure). Several authors, notably Elsasser [1951], Urey [1952] and Birch [1952], have put forward opposing arguments to the modified-utrabasic-rock core theory, but the opposing arguments themselves have been shown [Bullen 1952a, 1952b] to be subject to several weaknesses; the present writer's view is that it is still at least moderately probable that the region $E$ and the mantle are not sharply distinct chemically. In these circumstances, the original hypothesis (a) is possibly too broad a generalisation, so that it becomes necessary [Bullen 1950a, p. 58] to consider the less general hypothesis that:

b) a phase transition occurs at the boundary between the regions $D$ and $E$ of the Earth and leaves the compressibility and its pressure gradient unchanged on the two sides of the boundary.

At the same time it needs to be remarked that $k$, if determined by the relation $k / \rho=d p / d \rho$, would be discontinuous at the transition point itself, so that there are no $a$ priori physical grounds for expecting (b) to hold. Thus, on the emprical evidence stated in Section 3, (a) may well still be a fair approximation for a class of materials whose representative atomic number is close to that of the region $E$.

5. On the hypothesis (a), it is to be expected that the values of $k$ and $d k / d p$ in the regions $F$ and $G$ are close to those at the base of $E$. This would, however, be impossible on the results of Jeffreys for the $P$-velocity $\alpha$, if the inner core is fluid; (we ignore the extremely improbable happening that the density decreases with increase of depth in this part of the Earth). On the Jeffreys data, $\alpha$, increases suddenly at $r=1250 \mathrm{~km}$ by 15 per cent, so that if the inner core were composed of fluid for which $k=\rho \alpha^{2}$, there would be at least a 32 per cent jump in $k$ across the boundary. If, on the other hand, the inner core possesses significant rigidity $\mu$, with

$$
k+4 \mu / 3=\rho \alpha^{2},
$$

then $k$ will remain continuous across the boundary if $\mu$ has a value equal to about 25 per cent of that of $k$ in the inner core. The hypothesis (a), in conjunction with the data of Jeffreys, thus leads to the theory, stated in 1946 and developed in 1949 and $1950(8,9,18)$, that the Earth's inner core is solid in the sense that description of its elastic behaviour under stresses of periods of a few seconds requires the presence of a rigidity parameter of order $3.6 \times 10^{12} \mathrm{dyn} / \mathrm{cm}^{2}$. This conclusion has received support in further work of Ramsey [1950].

The diminution of $\alpha$ with increase of depth found by Jeffreys in the region $F$, in conjunction with the relation $k=\rho \alpha^{2}$, incidentally entails that, in $F$, either $\rho$ increases rapidly or else $k$ diminishes rapidly. On the hypothesis (a), the former alternative is preferred, the total increase in $\rho$ in $F$ being 25 per cent. Also the relatively low gradient of $\alpha$ inside $G$ entails that the average density gradient in $G$ is more than twice as great as the density gradient in the region $E$.

6. A consequence of rigidity in, the inner core is that the inner core could transmit $S$ seismic waves. On the basis of the Jeffreys data and the hypothesis (a), theoretical tables for a seismic phase PKJKP have been constructed [Bullen 1950b], where the symbol $J$ corresponds to $S$ waves in the inner core. Companion energy calculation [Bullen 1951] show, however, that such a phase could be only on the border of observability.

A comprehensive survey by Burke-Gaffney [Bullen and Burke-Gaffney 1952] of the whole series of seismograms recorded at the Riverview College Observatory between 1909 and1952 shows that none of these seismograms quite conforms to the theoretical requirements for a firm identification of the phase PKJKP, though a few observations could contribute to a wider statistical examination which would include data from a number of observatories. Further, the unknown character of the region $F$ leaves it possible that the energy in any $S$ waves generated in $G$ would be even less than the energy calculated [Bullen 1951] on the assumed boundary conditions. It follows that the rigidity of the inner core can at best be established by this direct seismic method only through intensive studies of seismograms followed by the use of searching statistical significance tests.

7. In the meantime, it is desirable to examine the sources of evidence for a solid inner core in some further detail. Points on which further investigation can usefully be made at present include:

i) a comparison of the effects of using Gutenberg's and Jeffreys' observational data on $\alpha$ in the inner core;

ii) questions arising from the forms that the compressibilitypressure hypothesis might take; 
iii) the bearing on the problem of quantum-mechanical data at extremely high pressures.

8. In considering (i), it will be sufficient to make an approximate calculation based on Gutenberg's velocity data quoted in Section 2.

We shall represent this data by

$$
\alpha^{2}=11.2^{2}-a(r-1000)
$$

where

$$
a=\left(11.2^{2}-10.1^{2}\right) / 250^{2},
$$

taking $d \alpha / d r$ to be zero at $r=1000 \mathrm{~km}$, and taking the $\mathrm{km}$ and sec as units. The equation (1) yields $0.19 \mathrm{~km} / \mathrm{sec}^{2}$ for the magnitude of $d \alpha \mu^{2} / d r$ when $r$ is just less than $1250 \mathrm{~km}$, as against $0.019 \mathrm{~km} / \mathrm{sec}^{2}$ when $r$ is just greater than $1250 \mathrm{~km}$, and implies that the magnitude of $d\{(k+4 \mu / 3) / \rho\} d r$ suddenly increases (as the depth increases) by a factor of ten at $r=1250 \mathrm{~km}$. On the hypothesis (a), it would follow that, while $\mu$ and its gradient are zero in the fluid region above $r$ $=1250 \mathrm{~km},|d(4 \mu / 3 \rho) / d r|$ changes to the value 0.17 $\mathrm{km} / \mathrm{sec}^{2}$ just below; i.e. $|d(\mu / \rho) d r|$ suddenly rises from zero to $0.13 \mathrm{~km} / \mathrm{sec}^{2}$. Assuming for $\beta^{2}(=\mu / \rho)$ a form similar to (1), we then obtain for $1000 \mathrm{~km}<r<1250 \mathrm{~km}$

$$
\beta^{2}=\beta_{\circ}^{2}-b(r-1000)^{2},
$$

where

$$
b=0.13 / 500 \text {, }
$$

and $\beta \circ$ is the $S$-velocity at $r=1000 \mathrm{~km}$. Since $\beta$ is here being taken as zero at $r=1250 \mathrm{~km}$, we then have $\beta_{\circ} \sim 4.0 \mathrm{~km} / \mathrm{sec}$ at $r=1000 \mathrm{~km}$.

Previous work using the data of Jeffreys had yielded $\beta_{\circ}=4.8 \mathrm{~km} / \mathrm{sec}$. The Jeffreys data had involved an increase in $\rho$ from 12 to $15 \mathrm{~g} / \mathrm{cm}^{3}$ in the region $F$ (see Section 5 ). If we ignore the possibility of an abnormal increase in density in the range $1000 \mathrm{~km}<r<1250 \mathrm{~km}$ (such increase is neither indicated nor precluded on Gutenberg's data), then the relation $\mu=\rho \beta^{2}$ would, on the hypothesis (a) and Gutenberg's data, yield a value of $\mu$ at $r=1000 \mathrm{~km}$ equal to $2.0 \times 10^{12} \mathrm{dyn} / \mathrm{cm}^{2}$, approximately, i.e. 0.6 of the value yielded on the Jeffreys data. If an abnormal density increase were present, the yielded rigidity would of course be greater. Thus, on the hypothesis (a) and Gutenberg's data, the minimum rigidity in the innermost $1000 \mathrm{~km}$ of the Earth appears to be about $2.0 \times 10^{12} \mathrm{dyn} / \mathrm{cm}^{2}$. This value is still comparable with the rigidity of the mantle, and is appreciably greater than that of surface rocks.

Between $r=1000 \mathrm{~km}$ and. the centre, Gutenberg's data would, on the hypothesis (a), yield a slightly higher density gradient than the Jeffreys data because of the less velocity gradient, but the density reached at the centre need not be much greater than $15 \mathrm{~g} / \mathrm{cm}^{3}$. This is closer to the figure preferred by Birch [1952], although Gutenberg's data do not preclude an appreciably higher value.

A further point is that whereas the main change in $\mu$ would take place suddenly on the Jeffreys data, it is only the gradient of $\mu$ that would change suddenly on the Gutenberg data, the change in $\mu$ being spread over $250 \mathrm{~km}$. The boundary conditions of the inner core would then be very different from those assumed in the energy calculations [Bullen 1951] for PKJKP, and the energy transformed into $S$ waves in the inner core would be very much less. Thus, if Gutenberg's velocity distribution is approximately correct, the phase PKJKP is quite unobservable, and it would not be possible to establish the rigidity of the inner core directly by observations of these waves.

9. The point (ii) of Section 7 on the possible limitations of the hypothesis (a) of Section 3 will now be considered in relation to the inner core.

If the view of Elsasser, Urey and Birch that the region $E$ consists of iron is substantiated, then the hypothesis (a) remains, as in 1946, an important working hypothesis. It is likely to be reliable at least to the extent that, for atomic numbers between about 18 and $29, k$ and $d k / d p$ at pressures of 1.3 million atmospheres differ by not more than the uncertainties in $k$ and $d k / d p$ in the writer's Earth model A [Bullen 1947, 1950a]. In Model A, the formal solution gave a reduction (not necessarily significant) of 5 per cent in $k$ at the boundary between the regions $D$ and E. Ramsey [Bullen 1949b; Ramsey 1950] prefers a greater change in $k$ at this boundary, but his argument involves deviation from homogeneity in the region $D$ to an extent that Birch [1952] regards as improbable. Ramsey [1950] finds $d k / d p$ for the mantle and core to be about the same, namely 3.7 and 3.53.8, respectively, while the writer's estimates yield 3.2-3.3 near the base of the mantle, and the same or a little less at the top of the core. Birch [1952], on independent grounds, finds the value in the mantle to be 3.3. In these circumstances, application of the hypothesis (a) to the inner core probably does not lead to errors in $k$ and $d k / d p$ of more than the order of 5 per cent.

On the assumption that the region $E$ consists of iron, and in the light of cosmic abundance data for elements, it is strongly probable that any deviation from the hypothesis (a) will involve changes of $k$ between $E$. and $G$ smaller than between $D$ and $E$. and therefore much smaller than would be required to bring the value of $\mu$ obtained in Section 5 down to zero. A 5 per cent jump in $k$ near the boundary of $G$ would still require the rigidity $\mu$ in the inner core to be nearly $3.1 \times 10^{12} \mathrm{dyn} / \mathrm{cm}^{2}$ on the Jeffreys data.

If, on the other hand, the region $E$ consists of modified ultrabasic rock, the remarks in Section 4 become relevant. 
On the view of Ramsey that the region $G$ is not chemically distinct from either $E$ or $D$, the expected change in $k$ between $E$ and $G$ would not be greater than between $D$ and $E$, and the argument favouring rigidity in $G$ is essentially unchanged. But the case favoured by the writer on present evidence [Bullen 1949c, 1950c, 1952a], in which $G$ consists of iron or denser materials while $E$ has the same chemical composition as the ultrabasic-rock material of $D$ is more difficult. The remark in the last paragraph of Section 4 shows that there is still some probability that the value of $k$ in $G$ is very close to that in the lower part of $E$, but a less general hypothesis than (a), closer to (b) of Section 4, would not lead of itself to any inference on the rigidity of $G$, and further considerations are desirable.

10. Such further considerations come from the point (iii) of Section 7; this supplements the argument in Section 9 in cases in which an estimate of the rigidity is yielded, and also goes some distance towards filling the gap in the more difficult case.

Several authors, including Jensen [1938], Feynman, Metropolis and Teller [1949], have considered the compressibility-pressure relations for Thomas-Fermi-Dirac gas models. Jensen, Elsasser [1951] and the writer [Bullen 1952b] have discussed the bearing of relevant parts of the results on the composition of the Earth's core. Elsasser quotes data at pressures of the order of 10 to 30 million atmospheres, according to which $k$ incre $_{1}$ ases by 8 per cent when the atomic number $Z$ changes from 18 to 36 . The pressure, $p_{1}$ say, at the boundary of the region $G$ is approximately 3.2 million atmospheres, and Elsasser's comparison of experimental results of Bridgman with the theoretical high-pressure results indicates about the same proportionate change of $k$ with $Z$ at the pressure $\mathrm{Pi}$ as at the higher pressures. The writer has amended certain parts of Elsasser's work, and finds that Bridgman's results, geophysical data and the Feynman data are well fitted together by taking smaller values of $k$ at the pressure $p_{1}$ than those assumed by Elsasser; this procedure raises the proportionate change in $k$ when $Z$ changes from 18 to 36 to about 12 per cent at the pressure $p_{1}$. Most theories of the composition of the Earth's core require the difference in $Z$ between the outer part of the region $G$ and the base of $E$ to be nearly zero; the writer's theory, referred to in Section 9, gives the biggest difference, of order seven units. Thus the quantum-mechanical calculations indicate that any jump in $k$ between $E$ and $G$ is of the order of 5 per cent or less, and that, on the Jeffreys velocity data, the rigidity of the inner core is still not less than the minimum value arrived at in Section 9. This argument is of course subject to uncertainties in the interpolations between Bridgman's data and the quantum-mechanical data, and again falls well short of deductive proof, but, in combination with the earlier discussion, leaves it most probable that the inner core is significantly rigid.

11. Gutenberg's data show appreciably smaller changes in $\alpha$ than Jeffreys' data in the vicinity of the boundary of the inner core, and may be reasonably taken as indicating the least allowable changes in $\alpha$. Taking then the lowest estimate of $\mu$ in Section 8 , diminished by a possible $0.5 \times 10^{12}$ $\mathrm{dyn} / \mathrm{cm}^{2}$ in accordance with the discussions in Sections 9 and 10 , we arrive at the value $1.5 \times 10^{12} \mathrm{dyn} / \mathrm{cm}^{2}$ as the least value of the rigidity in the inner core that is reasonably compatible with all present evidence. This value is less onehalf the value $3.6 \times 10^{12} \mathrm{dyn} / \mathrm{cm}^{2}$ involved in earlier calculations of the writer [Bullen 1950a, 1950b, 1951], but is still about twice the rigidity of steel at zero pressure.

As in earlier papers, temperature effects have been disregarded in all the foregoing discussions. Birch's recent work [1952] gives further confirmation that this would not lead to serious error.

If the rigidity of the inner core were in actual fact as low as the above suggested minimum value of $1.5 \times 10^{12}$ $\mathrm{dyn} / \mathrm{cm}^{2}$, there would be repercussions on the calculations of the travel-times of the phase PKJKP, and the time of the $J$ rays would need to be increased by a factor of 1.3 , approximately. This would entail increases ranging from zero up to about $2^{1 / 2}$ minutes in the calculated times. If the phase PKJKP arrived as late as this, however, the theoretical traveltime table [Bullen 1950b] would be devoid of practical value, since, on Gutenberg's data (see Section 8 ), the phase would not then be observable. But from the discussion in Sections 9 and 10 it follows that, even on the Jeffreys data, the times of the $J$ rays may need to be increased by a factor of 1.08, approximately. This would entail increases in the calculated times for $P K J K P$, reaching nearly 40 seconds at $\Delta=$ $180^{\circ}$.

It follows that, in seeking evidence on the occurrence of the phase $P K J K P$, it is desirable to consider readings on seismograms ranging up to perhaps a minute later than the times in the calculated table. The existence of the phase could be established only if sufficient of such readings fitted to adequate precision a travel-time curve possessing the right gradient. This consideration emphasises further that the existence of the phase PKJKP can be established only by statistical examination of a large number of readings.

\section{References}

Birch, F. (1952). Elasticity and constitution of the Earth's interior, J. Geophys. Res., 57, 227-286.

Bullen, K. E. (1940). The problem of the Earth's density variation, Bull. Seism. Soc. Amer., 30, 235-250.

Bullen, K. E. (1942). The density variation of the Earth's central core, Bull. Seism. Soc. Amer., 32, 19-29.

Bullen, K. E. (1946). A hypothesis on compressibility at 
pressures of the order of a million atmospheres, Nature, 157, 405.

Bullen, K. E. (1947). An introduction to the theory of seismology, Cambridge, Ch. XIII.

Bullen, K. E. (1949a). Compressibility-pressure hypothesis and the Earth's interior, M.N.R.A.S., Geophys. Suppl., 5, 355-368.

Bullen, K. E. (1949b). On the constitution of Venus, M.N.R.A.S., 109, 457-461.

Bullen, K. E. (1949c) On the constitution of Mars, M.N.R.A.S., 109, 688-692.

Bullen, K. E. (1950a). An Earth model based on a compressibilitypressure hypothesis, M.N.R.A.S., Geophys. Suppl., 6, 50-59.

Bullen, K. E. (1950b). Theoretical travel-times of $S$ waves in the Earth's inner core, M.N.R.A.S., Geophys. Suppl., 6, 125-128.

Bullen, K. E. (1950c). Venus and the Earth's inner core, M.N.R.A.S., 110, 256-259.

Bullen, K. E. (1951). Theoretical amplitudes of the seismic phase PKJKP, M.N.R.A.S., Geophys. Suppl., 6, 163-167.

Bullen, K. E. (1952a). Cores of terrestrial planets, Nature, 170, 363-364.

Bullen, K. E. (1952b). On density and compressibility at pressures up to thirty million atmospheres, Geophys. J. Int., 6, 383-401.

Bullen, K. E. and T. N. Burke-Gaffney (1952). Detection of $S$ waves in the Earth's inner core, Nature, 170, 455.

Elsasser, W. M. (1951). Quantum-theoretical densities of solids of extreme compression, Science, 113, 105-107.

Feynman, R. P., N. Metropolis and E. Teller (1949). Equations of state of elements based on the generalized FermiThomas theory, Phys. Rev., 75, 1561-1572.

Gutenberg, B. (1951). PKKP, P'P' and the Earth's core, Trans. Amer. Geophys. Union, 32, 373-390.

Gutenberg, B. and C. Richter (1938). P' and the Earth's core, M.N.R.A.S., Geophys. Suppl., 4, 363-372.

Gutenberg, B. and C. Richter (1939). On seismic waves, Gerlands Beitr. Geophys., 54, 94-136.

Jeffreys, H. (1939a). The times of the core waves, M.N.R.A.S., Geophys. Suppl., 4, 548-561.

Jeffreys, H. (1939b). The times of the core waves (second paper), M.N.R.A.S., Geophys. Suppl., 4, 594-615.

Jensen, H. (1938). Das Druck-Dichte Diagramm der Elemente bei höheren Drucken am Temperaturnullpunkt, Zeitsch. f. Physik, 111, 373-385.

Kronig, R., J. de Boer and J. Korringa (1946). On the internal constitution of the Earth, Physica, 12, 245-256.

Lehmann, I. (1936). P', Publ. Bur. Centr. Séism. Internat., série $A, 14,3-31$.

Ramsey, W. H. (1948). On the constitution of the terrestrial planets, M.N.R.A.S., 108, 406-413;

Ramsey, W. H. (1949). On the nature of the Earth's core, M.N.R.A.S., Geophys. Suppl., 5, 409-426.
Ramsey, W. H. (1950). On the compressibility of the Earth, M.N.R.A.S., Geophys. Suppl., 6, 42-49.

Urey, H. C. (1952). The planets, their origin and development, Yale.

C 2010 by the Istituto Nazionale di Geofisica e Vulcanologia. All rights reserved. 